\title{
Sífilis ocular: Presentación de diez casos y revisión de la literatura
}

\author{
Pablo Romero C., Cristhían Urzúa S., Patricia Gallardo V., Juan Verdaguer T., Marta Lechuga C., \\ Helio Hernández N., Felipe Sanhueza Z., Matías Andaur L. y Eugenio Donaire V.
}

\section{Ocular syphilis: Ten new cases and review of the literature}

Introduction: Ocular manifestations in patients with syphilis may involve almost any of the structures of the eye. Objectives: To describe ten new cases of syphilis with eye involvement and to briefly discuss the management and therapy of such condition. Material and Methods: Ten cases were retrospectively studied over 13 years. Demographic factors, ophthalmologic examination, and laboratory tests were assessed. Results: Seven of the 10 cases were male and 3 were female. The mean age of patients was 39,7 years. Disease presentation included: panuveitis (6 patients), optic neuritis (3), retinal vasculitis (1) and Argyll-Robertson pupil (1). Cerebrospinal fluid VDRL test was positive in 6 patients and 3 patients were HIV $(+)$. Conclusions: Syphilis is able to display diverse ophthalmologic manifestations. Not in all the cases the CSF-VDRL test was positive. Antitreponemal therapy generates a fast and effective response in the affected patients.

Key words: Ocular syphilis, Treponema pallidum, uveitis.

Palabras clave: Sífilis ocular, Treponema pallidum, uveítis. cientes con compromiso ocular por sífilis. Detallamos las manifestaciones clínicas y evolución posterior a la terapia específica destacando el alto índice de sospecha necesario para el diagnóstico, determinado por la ausencia de hallazgos patognomónicos de la enfermedad a nivel oftalmológico.

\section{Introducción}

$T$ reponema pallidum es el agente etiológico de la sífilis, enfermedad de transmisión sexual ${ }^{1,2}$. El compromiso del SNC se ve en 8 a $40 \%$ de los pacientes y puede ser: asintomático, manifestarse como un cuadro de meningitis o presentarse con tinnitus y/o vértigo $0^{3,4}$.

El compromiso ocular en general se presenta como una uveítis anterior que puede ser leve y asintomática en 5 a $10 \%$ de los casos, puede producir una reacción inflamatoria posterior, intermedia, anterior $\mathrm{y} / \mathrm{o}$ difusa ocular, uni o bilateral, granulomatosa o no granulomatosa ${ }^{5}$. Se ha descrito el compromiso de prácticamente cualquiera de las estructuras oculares ${ }^{6-9}$.

El diagnóstico se sospecha en presentaciones inusuales de un amplio abanico de enfermedades oculares, principalmente aquellas que afectan la úvea, confirmándose con la realización de tests treponémicos y no treponémicos en sangre y LCR.

En la serie de casos presentada se describen 10 pa-

\section{Pacientes y Métodos}

Se presentan diez nuevos casos provenientes de las consultas oftalmológicas del Hospital Clínico de la Universidad de Chile, recopilados entre agosto de 1995 y septiembre de 2008 .

Los parámetros evaluados fueron edad, sexo, agudeza visual mejor corregida-AVMC (letras en línea de la tabla de optotipos de Snellen) y hallazgos al examen oftalmológico como: examen de pupilas, observación en la lámpara de hendidura, medición de la presión intraocular-PIO normal menor de $21 \mathrm{~mm} \mathrm{Hg}$. Además se estimó la visión de colores (Test de Ishihara) ${ }^{10}$ y el examen de fondo de ojo.

También se evaluó clínicamente el compromiso del SNC y la respuesta oftalmológica al tratamiento. A todos los pacientes se les realizó VDRL en sangre y LCR previo y posterior al tratamiento, y pruebas treponémicas: FTAABS en sangre, previo tratamiento. Además a todos se les solicitó hemograma, VHS y serología (ELISA) para VIH. (tonómetro de aplanación de Perkins). Se consideró la PIO

\author{
Hospital Clínico Universidad \\ de Chile, Santiago. \\ Servicio de Oftalmología (PRC, CUS, \\ PGV, MLC, HHN). \\ Fundación Oftalmológica los Andes, \\ Santiago, Chile (JVT). \\ Clínica Río Blanco, Los Andes, Chile \\ Servicio de Urgencia (FSZ). \\ Universidad de Chile \\ Escuela de Medicina \\ Universidad de Chile, \\ Santiago. \\ Alumno Escuela de Pregrado (MAL, \\ EDV). \\ Recibido: 8 de abril de 2010 \\ Aceptado: 27 de septiembre de \\ 2010 \\ Correspondencia a: \\ Pablo Romero Carrasco \\ pablotromero@med.uchile.cl
}


En algunos casos además se realizó resonancia magnética o TAC de cerebro, concentraciones plasmáticas de vitamina B-12, serología para toxoplasmosis, campo visual de Goldmann y exámenes inmunológicos (factor reumatoídeo, ANA, ANCA y ENA).

\section{Resultados}

Se evaluaron 10 pacientes sin diagnóstico previo de sífilis, 7 hombres y 3 mujeres, con edades entre los 21 y 56 años; la edad promedio fue 39 años. Los casos fueron diagnosticados entre los años 1995 y 2008 . Como muestra el Gráfico 1, la mayor cantidad de los casos fueron detectados en los últimos cinco años.

La Tabla 1 muestra un resumen de los hallazgos clínicos y demográficos encontrados en el grupo estudiado.

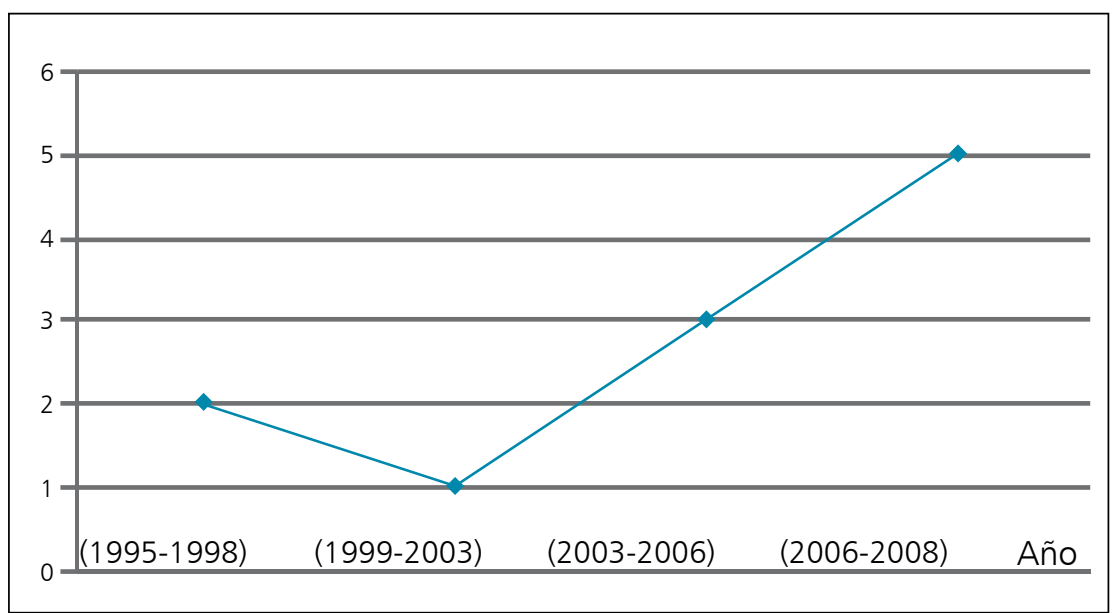

Gráfico 1. Relación entre el número de casos y año.
Las presentaciones clínicas observadas fueron: panuveítis en 6 pacientes, neuritis óptica en 3, vasculitis retinal en 2 y pupila de Argyll-Robertson en un paciente cada una. El compromiso bilateral se observó en 9 pacientes $(90 \%)$. La agudeza visual (AV) previa a tratamiento fue $\leq 0,1$ en la mayoría de $\operatorname{los}_{\text {casos }}{ }^{9}$ y sólo $10 \%$ tenía AV mayor de 0,67 al inicio del tratamiento.

Las pruebas no treponémicas, mostraron VDRL en sangre $(+)$ en todos los pacientes y en LCR $(+)$ en seis, siendo (-) en cuatro pacientes. En uno de los cinco pacientes con panuveitis el VDRL en LCR resultó negativo, también lo fue en uno de los tres pacientes con neuritis óptica, en uno de dos con vasculitis retinal y en el paciente que presentaba pupila de Argyll-Robertson.

Todos los pacientes fueron tratados con penicilina $G$ sódica acuosa 18 a 24 millones U.I. e.v. al día, durante catorce días, hospitalizados, seguida de 2,4 millones de U.I. semanales de penicilina $\mathrm{G}$ benzatina im, durante 3 semanas ${ }^{11}$

La respuesta al tratamiento fue categórica en todos los casos. La AV mejoró en 80\% (n: 8). No mejoró en dos de los tres pacientes con neuritis óptica. En todos los pacientes la RM de cerebro fue normal.

A continuación se describen los casos en forma individual.

\section{Caso 1: Vasculitis retinal y panuveitis}

Hombre de 45 años con el antecedente de gonorrea genital tratada 15 años antes, sin otros antecedentes mórbidos. Consultó por disminución progresiva de la AV que se habría iniciado dos años antes, mayor en el ojo derecho, asociada a dolor ocular ocasional bilateral y cefalea intermitente.

\begin{tabular}{|c|c|c|c|c|c|c|c|c|c|}
\hline \multirow{2}{*}{$\begin{array}{c}\mathbf{n} \\
1\end{array}$} & \multirow{2}{*}{$\begin{array}{c}\text { Edad } \\
\text { (años) } \\
45\end{array}$} & \multirow{2}{*}{$\begin{array}{c}\text { Género } \\
\text { M }\end{array}$} & \multicolumn{2}{|c|}{ AVMC previo a tratamiento } & \multicolumn{2}{|c|}{ AVMC posterior a tratamiento } & \multirow{2}{*}{$\begin{array}{c}\text { VDRL } \\
\text { en LCR } \\
(-)\end{array}$} & \multirow{2}{*}{$\begin{array}{l}\text { VIH } \\
(-) \\
\end{array}$} & \multirow{2}{*}{$\begin{array}{c}\text { Diagnóstico } \\
\text { Vasculitis retinal y panuveiti }\end{array}$} \\
\hline & & & OD: cd a $1 \mathrm{mt}$ & Ol: 0,5 & OD: cd a $1 \mathrm{mt}$ & Ol: 0,8 & & & \\
\hline 2 & 27 & M & OD: 0,05 & OI: 0,1 & OD: 0,67 & OI: 0,67 & $(-)$ & $(-)$ & Neuritis óptica bilateral \\
\hline 3 & 58 & $\mathrm{~F}$ & OD: 1,0 & Ol: 1,0 & OD: 1,0 & Ol: 1,0 & $(-)$ & $(-)$ & Pupila de Argyll Robertson \\
\hline 4 & 45 & $\mathrm{~F}$ & OD: 0,05 & OI: 0,05 & OD: 0,05 & OI: 0,05 & $(+)$ & $(-)$ & Neuritis óptica bilateral \\
\hline 5 & 39 & M & OD: 0,1 & Ol: $\mathrm{cd}$ a $1 \mathrm{mt}$ & OD: 0,7 & Ol: 0,5 & $(-)$ & $(-)$ & Panuveitis \\
\hline 6 & 34 & M & OD: 0,05 & OI: 0,1 & OD: 0,2 & OI: 0,3 & $(+)$ & $(-)$ & Neuritis óptica bilateral \\
\hline 7 & 45 & M & OD: 0,1 & Ol: 0,1 & OD: 0,9 & Ol: 0,9 & $(+)$ & $(+)$ & Panuveitis \\
\hline 8 & 27 & M & OD: $\mathrm{mm}$ a $1 \mathrm{mt}$ & OI: $\mathrm{mm}$ a $1 \mathrm{mt}$ & OD: 1,0 & OI: 1,0 & $(+)$ & $(+)$ & Panuveitis \\
\hline 9 & 21 & M & OD: $\mathrm{mm}$ a $1 \mathrm{mt}$ & OI: 0,4 p & OD: 0,8 & Ol: 1,0 & $(+)$ & $(+)$ & Panuveitis \\
\hline 10 & 56 & $\mathrm{~F}$ & OD: $c d$ a $1 \mathrm{mt}$ & Ol: $\mathrm{cd}$ a $1 \mathrm{mt}$ & OD: cd a $1 \mathrm{mt}$ & OI: cd a $1 \mathrm{mt}$ & $(+)$ & $(-)$ & Panuveitis y vasculitis retinal \\
\hline
\end{tabular}


Al examen oftalmológico presentaba AVMC en el ojo derecho (OD) igual a cuenta dedos a 1 metro y 0,5 en el ojo izquierdo (OI). Defecto pupilar aferente relativo (DPAR) presente en el ojo derecho. Al examen al biomicroscopio presentaba Tyndall +++ en el OD y ++ en el OI. PIO igual a $34 \mathrm{~mm} \mathrm{Hg}$ en el OD y $29 \mathrm{~mm} \mathrm{Hg}$ en el OI.

Al examen del fondo de ojo destacaba edema de papila bilateral, vitreítis posterior mayor en el OD, hemorragias pre-retinales, neo-vascularización retinal bilateral y cicatriz macular en el OD.

Los exámenes de laboratorio mostraron VDRL en sangre (+), título: 1/128, FTA-ABS (+) y VDRL en el LCR (-). El estudio citoquímico del LCR resultó normal, PPD igual a $24 \mathrm{~mm}$, serología para VIH y Toxoplasma gondii (-) y una TAC de cerebro sin hallazgos patológicos.

Se diagnosticó una vasculitis retinal bilateral y panuveítis. Se trató con el esquema de penicilina antes indicado con evolución favorable, desapareciendo todos los signos inflamatorios a las dos semanas de iniciado el tratamiento. La AVMC mejoró a 0,8 en el OI; el OD no mejoró.

\section{Caso 2: Panuveitis y neuritis óptica bilateral}

Hombre de 27 años, sin antecedentes mórbidos. Luego de estar consumiendo drogas (clorhidrato de pasta base) notó disminución de la AV bilateral y ojos rojos. El cuadro se acompañó de cefalea frontal y fotopsias bilaterales. Consultó una semana después.

Al examen oftalmológico destacaba: AVMC en el OD igual a 0,05 y en el OI igual a 0,1. Visión de colores alterada en ambos ojos. Reflejos pupilares lentos bilaterales y sin DPAR. PIO igual a $24 \mathrm{~mm} \mathrm{Hg}$ en el OD y $23 \mathrm{~mm} \mathrm{Hg}$ en el OI. Al examen al biomicroscopio destacaba Tyndall +++ en ambos ojos. Presentaba al fondo de ojo vitreítis ++ bilateral. Ambas papilas estaban levemente pálidas. El campo visual de Goldmann mostró escotomas centro centrales bilaterales (Figura 1).

La TAC de cerebro no mostró hallazgos patológicos. Se planteó el diagnóstico de neuritis óptica bilateral y panuveitis. El estudio etiológico mostró VDRL en sangre (+), título: 1/128, FTA-ABS (+) y VDRL en LCR (-). El estudio citoquímico del LCR resultó normal. Se realizó tratamiento con penicilina según pautas. Diez días después de iniciada la terapia los campos visuales mostraron una importante mejoría y la agudeza visual mejoró a 0,67 en ambos ojos (Figura 2).

\section{Caso 3: Pupila de Argyll Robertson}

Mujer de 58 años con el antecedente de hipotiroidismo post tiroidectomía efectuada por hiperplasia tiroidea multinodular, en tratamiento con levotiroxina. Sin antecedente de sífilis genital. Consultó para cambio de lentes de cerca. Al examen destacaba una AVMC igual a 1,0 en ambos ojos. Visión de colores normal en ambos ojos. Ambas pupilas mióticas. Reflejo fotomotor directo y consensual presentes, disociación luz-cerca positiva y sin DPAR. El examen al biomicroscopio y el fondo de ojo resultaron normales.

Los exámenes de laboratorio mostraban VDRL en sangre (+), título 1/32 y FTA-ABS (+), VDRL en LCR (-). El estudio citoquímico del LCR resultó normal. Una RM cerebral fue normal. Se diagnosticó pupila de Argyll Robertson. Se hospitalizó realizándosele tratamiento con el esquema habitual de penicilina. A pesar del tratamiento antimicrobiano los reflejos pupilares permanecieron iguales.

\section{Caso 4: Neuritis óptica}

Mujer de 45 años que consultó por cuadro de disminución progresiva de la AV bilateral con evolución de dos años. Tenía el antecedente de una atrofia óptica bilateral en estudio, sin antecedente de sífilis genital. Al examen destacaba una AVMC igual a 0,05 en el OD y 0,05 en el OI. PIO igual a $18 \mathrm{~mm} \mathrm{Hg}$ en el OD y $15 \mathrm{~mm} \mathrm{Hg}$ en el OI. Pupilas isocóricas. Reflejos pupilares enlentecidos, con DPAR $(+)$ en el OD.

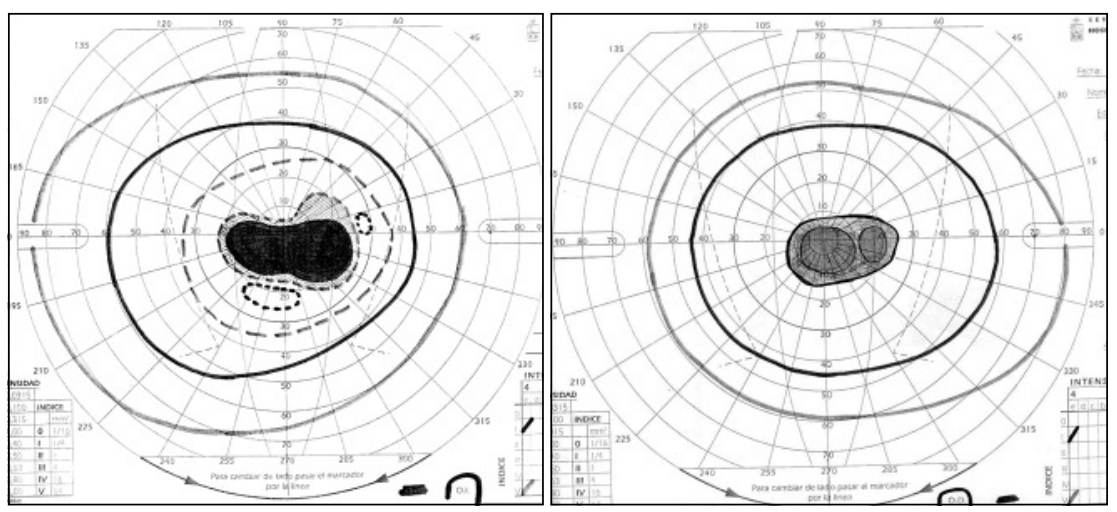

Figura 1. Campo visual de Goldmann previo tratamiento muestra escotomas centrocecales en ambos ojos.
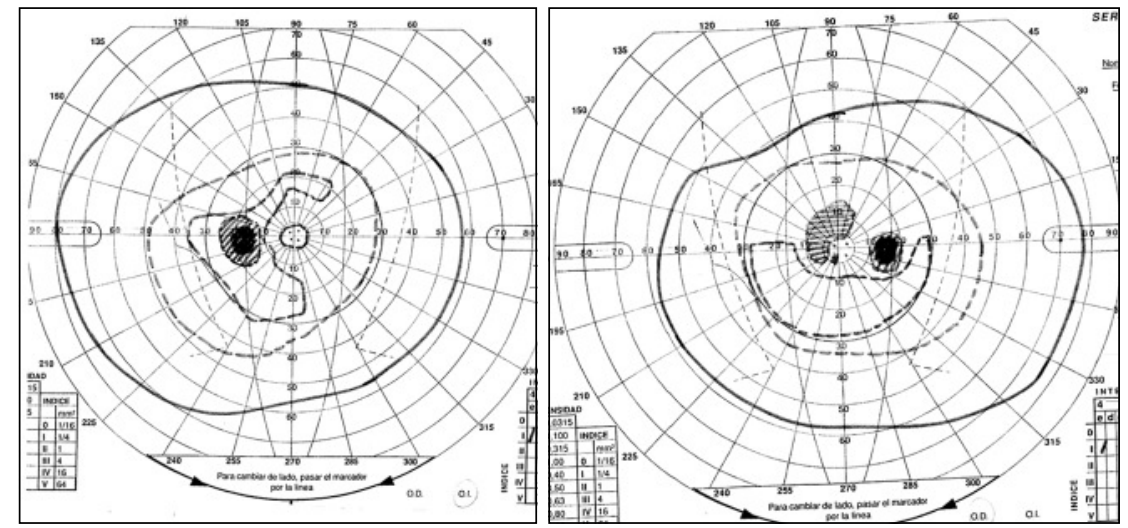

Figura 2. Campo visual post tratamiento muestra franca mejoría. 
El examen del fondo de ojo destacaba papilas pálidas, bordes netos, excavación 0,3 bilateral. Los campos visuales mostraban estrechamiento concéntrico bilateral.

Los exámenes de laboratorio arrojaron VDRL en sangre $(+)$, título: 1/128 y FTA-ABS (+) y VDRL en LCR $(+)$. RM normal. Se diagnosticó una neuritis óptica bilateral y se le realizó tratamiento con penicilina en esquema habitual. Evolucionó con detención del daño funcional, manteniéndose la AVMC en 0,05 en ambos ojos al año de haberse tratado, sin modificaciones de los campos visuales.

\section{Caso 5: Panuveítis bilateral}

Hombre de 39 años con el antecedente de uveítis reactiva bilateral desde hacía un año, en tratamiento con prednisona $20 \mathrm{mg} /$ día y metotrexato. Úlceras bucales y anales y portador de virus de hepatitis B. Refería un rash en las rodillas y descamación de palmas y plantas desde hacía un mes, asociado a persistencia de sintomatología oftalmológica consistente en fotofobia, ojo rojo y disminución de la AV bilateral.

Al examen oftalmológico destacaba una AVMC de 0,1 en el OD y cuenta dedos a $30 \mathrm{~cm}$ en el OI. Pupilas isocóricas reactivas y simétricas y sin DPAR. PIO igual a $25 \mathrm{~mm} \mathrm{Hg}$ en el OD y $24 \mathrm{~mm} \mathrm{Hg}$ en el OI. Al examen al biomicroscopio se observaba una cornea clara, cámara anterior amplia y Tyndall ++ bilateral, sin sinequias.

En el fondo de ojo se observaban papilas planas, edema de mácula bilateral, vasos adelgazados y tortuosos sin envainamiento. Los exámenes de laboratorio mostraron: VDRL en sangre (+), títulos: 1/128, FTA-ABS (+), VDRL en LCR (-), ANCA (-), VHS: 97, ANA (+) y PCR: 71,9 $\mathrm{mg} / \mathrm{L}$. ELISA para VIH (-). Se diagnosticó una panuveítis. Se trató con penicilina en forma habitual, evolucionando con disminución de la inflamación ocular y franca mejoría de la AV (OD: 0.67 y OI: 0.5 )

\section{Caso 6: Neuritis óptica bilateral}

Hombre de 34 años, con el antecedente de visión borrosa hacía ya seis meses, sin antecedentes de sífilis. Al examen oftalmológico destacaba AVMC de 0,025 en el OD y 0,05 en el OI. Pupilas isocóricas reactivas y simétricas. Disociación luz-cerca positiva, con DPAR $(+)$ en el OD.

El examen al biomicroscopio mostraba una cornea clara, cámara anterior amplia y cristalino claro. En el fondo de ojo se observaban papilas pálidas, excavación 0,8 en el OD y 0,4 en el OI. Los exámenes de laboratorio revelaron concentraciones de vitamina B-12 normales, VDRL en sangre $(+)$, título 1/32, FTA-ABS (+), VDRL en LCR (+), ELISA para VIH (-). RM cerebral normal. Campo visual de Goldmann mostró un defecto altitudinal bilateral (Figura 5).

Se diagnosticó una neuritis óptica bilateral luética. Se hospitalizó e indicó tratamiento con penicilina en esquema habitual. Evolucionó con mejoría de la AV (AVMC: 0,2 en el OD y 0,3 en el OI) y del campo visual al mes de iniciado el tratamiento.

\section{Caso 7: Panuveítis bilateral}

Hombre de 45 años edad, con el antecedente de ser portador de VIH, sin terapia anti-retroviral y sin antecedente de sífilis genital. Consultó por cuadro que evolucionaba hacían tres semanas, caracterizado por ojo rojo bilateral, mayor en OD.

$\mathrm{Al}$ examen oftalmológico presentaba AVMC igual a 0,05 en el OD y 0,1 en el OI. PIO de $27 \mathrm{~mm} \mathrm{Hg}$ en el OD y $25 \mathrm{~mm} \mathrm{Hg}$ en el OI. Al examen al biomicroscopio presentaba hiperemia conjuntival bilateral, cornea con precipitados queráticos pequeños, mayor en el OD, sinequias posteriores en el OI, Tyndall ++ en el OD y + en el OI (Figura 6). Al examen del fondo de ojo destacaba vitreítis bilateral, papila rosada, bordes netos, excavación de 0,3 bilateral.

Los exámenes de laboratorio mostraron VDRL en sangre $(+)$, título 1/32, VDRL en LCR $(+)$, estudio citoquímico de LCR normal, FTA-ABS $(+)$. TAC de cerebro sin hallazgos patológicos. Se diagnosticó una panuveitis bilateral por sífilis y se le practicó tratamiento con esquema habitual de penicilina. Evoluciono con disminución de la inflamación bilateral a las dos semanas de iniciado el tratamiento y mejoría de la AV.

\section{Caso 8: Panuveítis bilateral}

Hombre de 27 años, sin antecedente de sífilis genital ni otros antecedentes mórbidos. Consultó por disminución progresiva de la AV bilateral que se habría iniciado dos meses antes, asociada a dolor ocular bilateral. Había recibido tratamiento durante siete semanas con corticoides tópicos y sistémicos pese a lo cual hubo empeoramiento progresivo del cuadro.

Presentaba AVMC igual a movimiento de manos en ambos ojos. PIO igual a $29 \mathrm{~mm} \mathrm{Hg}$ en el OD y $31 \mathrm{~mm} \mathrm{Hg}$ en el OI. Al examen al biomicroscopio presentaba Tyndall +++ en ambos ojos, sinequias posteriores periféricas. $\mathrm{Al}$ examen del fondo de ojo destacaba vitreítis bilateral intensa que impedía ver detalles.

Los exámenes de laboratorio mostraron VDRL en sangre $(+)$, título: $1 / 32$, VDRL en LCR $(+)$. Estudio citoquímico de LCR normal, FTA-ABS $(+)$. Serología ELISA para VIH (+) y para Toxoplasma gondii (-). TAC de cerebro sin hallazgos patológicos.

Se diagnosticó una panuveitis bilateral y se le realizó tratamiento con penicilina según el esquema habitual. Evolucionó con disminución de la inflamación bilateral a las dos semanas de iniciado el tratamiento y mejoría de la AV. 


\section{Caso 9: Panuveitis bilateral}

Hombre de 25 años previamente sano, quien consultó por disminución de la $\mathrm{AV}$ del $\mathrm{OD}$, asociado a ojo rojo y fotofobia que se habría iniciado dos días previos. Acudió a un centro oftalmológico donde se le diagnosticó uveítis anterior en el OD e inició tratamiento con corticoides tópicos y midriáticos. Evolucionó desfavorablemente agregándose mayor disminución de la AV y dolor ocular bilateral. Consultó tres semanas después del inicio de la sintomatología al Hospital Clínico de la Universidad de Chile.

$\mathrm{Al}$ examen oftalmológico presentaba AVMC igual a movimiento de manos a 1 metro en el OD y 0,4 parcial en el OI. Reflejos pupilares y disociación luz-cerca no evaluables por tratamiento con atropina. PIO igual a 23 $\mathrm{mm} \mathrm{Hg}$ en el OD y $26 \mathrm{~mm} \mathrm{Hg}$ en el OI. Al examen al biomicroscopio presentaba Tyndall +++ en el OD y ++ en el OI. Sinequias posteriores bilaterales mayor en el OD, precipitados queráticos en el OI, roséola sifilítica en el OD y nódulo de Busacca en el OD. PIO de aplanación igual a $11 \mathrm{~mm} \mathrm{Hg}$ en el OD y $18 \mathrm{~mm} \mathrm{Hg}$ en el OI.

$\mathrm{Al}$ examen del fondo de ojo destacaba una vitreítis bilateral mayor en el OD, foco de coroiditis en el OD y vasos dilatados.

El estudio de laboratorio mostró un hemograma sin leucocitosis ni formas inmaduras (desviación a izquierda), VDRL en sangre $(+)$ : titulo $1 / 16$, VDRL en LCR $(+)$ 1:4 estudio citoquímico de LCR normal, FTA-ABS $(+)$, PPD (+): $24 \mathrm{~mm}$. Serología para Toxoplasma gondii (-) y ELISA para VIH (+). El estudio para virus de hepatitis B (HBsAg) y virus de hepatitis C (ELISA) fue negativo. TAC de cerebro sin hallazgos patológicos,

Se diagnosticó una panuveítis bilateral y se realizó tratamiento con penicilina según el esquema habitual. Evolucionó con disminución de la inflamación bilateral a las dos semanas de iniciado el tratamiento y mejoría de la agudeza visual en ambos ojos 0,8 en el OD y 1,0 en el OI.

\section{Caso 10: Panuveítis bilateral y vasculitis}

Mujer de 56 años derivada desde la ciudad de La Serena, con los antecedentes de eritema nodoso en estudio y retinitis por citomegalovirus (CMV) desde hacía al menos dos años. Consultó con cuadro de disminución de la AV de dos meses de evolución. Con VDRL en sangre (-) un año antes.

Al examen oftalmológico destacaba una AV de cuenta dedos en ambos ojos y tonometría (PIO) normal. Ambos ojos presentaban Tyndall + , precipitados queráticos en grasa de carnero bilateral, sinequias posteriores, que impedía dilatar ambas pupilas, facodonesis y Tyndall vítreo. Al examen del fondo de ojo se observaban focos de actividad de aspecto algodonoso, perivasculitis, zonas de atrofia retiniana bilaterales y placas de corio-retinitis bilaterales (Figura 3).

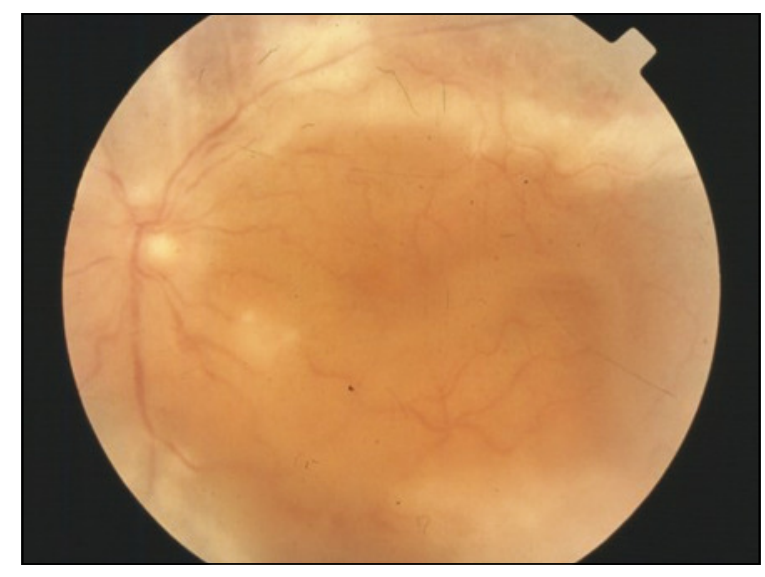

Figura 3. Fondo de ojo previo al tratamiento. Perivasculitis y placas gruesas de corioretinitis bilaterales.

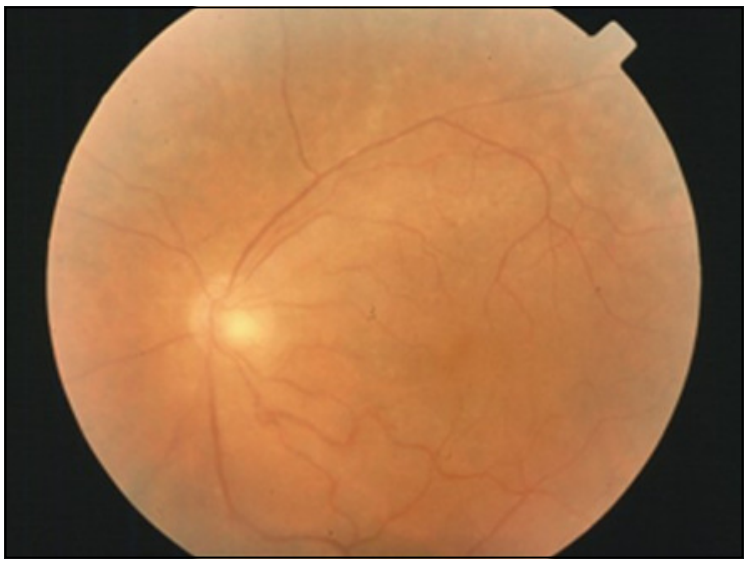

Figura 4. Fondo de ojo cuatro semanas posterior al tratamiento muestra franca disminución de la inflamación.

Se inició tratamiento para retinitis por CMV con ganciclovir $5 \mathrm{mg} / \mathrm{kg} /$ día, sin mejoría. Los nuevos exámenes de laboratorio mostraron: VDRL en sangre $(+)$, título: 1:256, FTA-ABS $(+)$, VDRL en LCR $(+) 1 / 4$. Serología para CMV (-) y para VIH (-), VHS=30, PCR: $6 \mathrm{mg} / \mathrm{L}$ y ANCA (-).

Se diagnosticó una panuveítis bilateral y vasculitis y se le realizó tratamiento con dosis habituales de penicilina. Evolucionó con disminución de la inflamación bilateral a las dos semanas de iniciado el tratamiento (Figura 4) y mejoría de la visión.

\section{Discusión}

El compromiso ocular secundario a sífilis puede presentarse de variadas formas clínicas ${ }^{5,8}$, por eso se le conoce como "la gran simuladora". La uveítis es la manifestación más común de la sífilis, tanto en los pacientes con coinfección por VIH como sin ella ${ }^{5,12}$. Las presentaciones 
clínicas encontradas en nuestro estudio coinciden con estas afirmaciones: panuveítis (6 pacientes), neuritis óptica (3), vasculitis retinal (2), y pupila de Argyll-Robertson. Esto nos muestra que debemos considerar el diagnóstico de sífilis ocular frente a cuadros inflamatorios poco frecuentes, especialmente panuveítis bilateral atípicas y neuroretinitis.

En todos los pacientes encontramos VDRL reactivo en sangre. Los valores de los títulos fluctuaron entre 1/16 y $1 / 256$. Tal como han mencionado otros autores, en los pacientes con sífilis ocular el VDRL en LCR no siempre resulta positivo ${ }^{8,11,13}$. En nuestra serie el VDRL en LCR resultó (-) en $40 \%$ de los casos. En todos los pacientes co-infectados con VIH el VDRL en LCR resultó $(+)$. A pesar de no haber realizado una prueba estadística, por la pequeña y variada muestra de los pacientes, no observamos alguna relación entre el tipo de patología ocular y la positividad de VDRL en LCR.

Los títulos de VDRL en LCR son variables; incluso, en pacientes con neurosífilis estos pueden ser menores de $1 / 32$, dependiendo del grado de inmunosupresión ${ }^{14}$. De los 6 pacientes que presentaban VDRL $(+)$ en el LCR, sólo tuvimos acceso a los títulos en dos de ellos, cuyos valores fueron iguales: 1:4.

Además de las pruebas treponémicas y no treponémicas en sangre y LCR, existen otros métodos diagnósticos también efectivos pero más invasores como es la detección de Treponema pallidum en el vítreo por $\mathrm{RPC}^{15,16}$. Creemos que tanto las pruebas treponémicas de las que disponemos en la actualidad, así como la clara respuesta al tratamiento, son suficientes para realizar un diagnóstico certero. La demora en la solicitud de pruebas treponémicas puede retrasar el diagnóstico y el inicio de un tratamiento adecuado. Existen numerosos nuevos tests diagnósticos para sífilis tales como: ensayos de quimioluminiscencia y enzimas de inmunoensayos, entre otras, las que aún no se usan de forma habitual para la identificación de $T$. pallidum, pero podrían tener un valor en el futuro ${ }^{17}$.

Es importante destacar que la uveítis asociada a sífilis produce complicaciones que revelan la agresividad del cuadro, como son la presencia de sinequias posteriores, que pueden llevar a la producción de catarata y glaucoma. Esto refuerza la importancia del estudio oportuno y del tratamiento de la enfermedad de base. A pesar de la poca respuesta anti-inflamatoria en ausencia del tratamiento antimicrobiano sistémico, se recomienda el tratamiento agresivo local con anti-inflamatorios tópicos y midríaticos para evitar las complicaciones oculares que se pueden presentar $^{18}$.

El tratamiento de la sífilis ocular debe incluir además una evaluación multidisciplinaria con el objeto de buscar manifestaciones extra-oculares y tratar otras patologías asociadas. Dado que las manifestaciones oculares de la sífilis con frecuencia se asocian con neurosífilis está reco- mendado realizar examen del LCR a todos los pacientes.

La sífilis ocular debe ser tratada como neurosífilis con penicilina $\mathrm{G}$ sódica acuosa ev 18 a 24 millones UI/día, durante 10 a 14 días ${ }^{8,11,19,20}$. Posteriormente, según la opinión de expertos, se recomienda el uso de penicilina benzatina, 2,4 millones UI semanal im durante tres semanas ${ }^{18}$. La duración del tratamiento aún es una controversia, siendo para algunos autores suficiente emplear dos semanas ${ }^{8,5,21}$; las guías norteamericanas de manejo de ETS recomiendan 10 a 14 días $^{22}$.

Como alternativa terapéutica, pacientes con buena adherencia se pueden manejar con penicilina procaína 2,4 millones UI /día IM asociado a probenecid 2 gramos cada día, durante 10 a 14 días, lo que determina ventajas en relación a la administración ambulatoria y la reducción de los costos asociado a esto ${ }^{18,20}$.

Los pacientes genuinamente alérgicos a penicilina podrían ser tratados con ceftriaxona ( 2 gramos al día durante 14 días), aunque existe la posibilidad de una reacción cruzada con la cefalosporina por lo que los pacientes alérgicos deben ser idealmente evaluados, desensibilizados y manejados por especialista ${ }^{20,23}$.

Doxiciclina, tetraciclina y azitromicina oral han sido estudiadas como terapia alternativa para la sífilis primaria, en personas alérgicas a penicilina o en regiones del mundo donde el acceso a penicilina o la administración im o ev de este medicamento sean difíciles ${ }^{24-26}$. Sin embargo, estos estudios fueron hechos en pacientes con sífilis temprana y no han sido evaluados adecuadamente para el tratamiento de la sífilis secundaria o terciaria.

En esta serie clínica, en todos los pacientes el tratamiento específico anti-treponémico, en dosis adecuadas, produjo una rápida y efectiva respuesta, con una clara mejoría a los pocos días de iniciado el tratamiento. Observamos que los pacientes con mejor pronóstico visual fueron aquellos con uveítis. El cuadro asociado a mala visión fue la neuritis óptica; esto puede explicarse por el hecho de que en ella debemos actuar de forma oportuna antes de que se produzca la atrofia óptica irreversible.

En pacientes co-infectados con VIH, la sífilis es la causa más frecuente de infección bacteriana intraocular ${ }^{27,28}$. Se presenta como una iridociclitis o inflamación intraocular difusa, neuritis óptica o retinitis ${ }^{29}$. Los diagnósticos diferenciales de retinitis en pacientes con infección por $\mathrm{VIH}$ son la retinitis por CMV, toxoplasmosis ocular, y la retinitis herpética necrosante que incluye las retinitis producidas por otros virus de la familia herpes: herpes simplex y varicela-zoster ${ }^{30}$. La retinitis sifilítica es menos frecuente que las anteriores, y en estos casos es importante evaluar la presencia de lesiones cutáneo-mucosas que pueden facilitar el diagnóstico ${ }^{28,31}$.

En todos los pacientes con sífilis ocular se debe descartar la presencia de infección por VIH, ambas enfermedades comparten los mismos factores de riesgo, 
pudiendo estar ambas presentes ${ }^{5,32,33}$. La sífilis ocular puede ser más grave en pacientes infectados por VIH que no estén recibiendo terapia anti-retroviral ${ }^{8,28}$. Nosotros observamos una co-infección sífilis/VIH de 30\%. Todos los casos en que se obtuvo serología positiva para VIH correspondían a pacientes con uveítis. Las PIOs estaban elevadas en $86 \%$ de aquellos pacientes con uveítis, como ya ha sido descrito en otras publicaciones ${ }^{34}$.

En el caso de la pupila de Argyll Robertson el tratamiento no modificó los reflejos pupilares; esto podría deberse a que las lesiones en las células de la región pretectal del mesencéfalo, secundarias a la neurolúes son irreversibles y las señales procedentes de la retina no son transmitidas por el núcleo pretectal a los núcleos de Edinger-Westphal, produciendo la pérdida permanente de la reacción pupilar a la luz directa y consensual ${ }^{35}$.

La prevalencia de sífilis se ha incrementado notablemente en los últimos cinco años en el mundo ${ }^{8,19}$. Nosotros observamos que gran parte de los casos enrolados se diagnosticaron en los últimos ocho años.

Esta experiencia clínica pretende reforzar que la sífilis ocular es una entidad aún presente, pudiendo tener diversas manifestaciones, así como la importancia de la búsqueda activa de ella, en casos similares a los mostrados en este trabajo. Además destacar que es una enfermedad potencialmente grave que afecta a adultos jóvenes, con buena respuesta a tratamiento si el diagnóstico se realiza de manera precoz y la importancia de la búsqueda del binomio infección por VIH-sífilis.

\section{Resumen}

Introducción: Las manifestaciones oculares de los pacientes con sífilis pueden comprometer cualquiera de las estructuras del ojo. Objetivos: Describir diez nuevos casos de sífilis con compromiso ocular y realizar una breve discusión de su manejo y tratamiento. Pacientes y Métodos: Se estudiaron, de forma retrospectiva, diez casos en un período de 13 años. Se evaluaron factores demográficos, exámenes oftalmológico y de laboratorio. Resultados: De los diez casos, 7 eran hombres y 3 mujeres. El promedio de edad fue de 39,7 años. Las presentaciones fueron: panuveítis (n: 6), neuritis óptica (n: 3), vasculitis retinal (n: 1) y pupila de Argyll-Robertson (n: 1). Se obtuvo VDRL $(+)$ en líquido cefalorraquídeo en 6 pacientes y serología para $\mathrm{VIH}(+)$ en 3 pacientes. Conclusiones: La sífilis puede producir variadas manifestaciones oftalmológicas. No en todos los casos el VDRL resultó positivo en el LCR. El tratamiento anti-treponémico produce una rápida y efectiva respuesta en los pacientes afectados.

\section{Referencias}

1.- Finelli L, Levine W C, Valentine J, St Louis M E. Syphilis outbreak assessment. Sex Transm Dis 2001; 28 (3): 131-5.

2.- Kent M E, Romanelli F. Reexamining syphilis: An update on epidemiology, clinical manifestations, and management. Ann Pharmacother 2008; 42 (2): 226-36.

3.- Rivera E, Codjambassis L, Cabello F. Neurosífilis: estudio de 19 casos en Valparaíso, Chile. Rev. Chil. Neuro-psiquiatr 2002; 40: 253-7.

4.- Zaidman G W. Neurosyphilis and retrobulbar neuritis in a patient with AIDS. Ann Ophthalmol 1986; 18 (9): 260-1.

5.- Aldave A J, King J A, Cunningham E T Jr. Ocular syphilis. Curr Opin Ophthalmol 2001; 12 (6): 433-41.

6.- Kiss S, Damico F M, Young L H. Ocular manifestations and treatment of syphilis. Semin Ophthalmol 2005; 20 (3): 161-7.

7.- Wilhelmus K R, Jones D B. Adult-onset syphilitic stromal keratitis. Am J Ophthalmol 2006; 141 (2): 319-21.

8.- Gaudio P A. Update on ocular syphilis. Curr Opin Ophthalmol 2006; 17 (6): 562-6.

9.- Fonollosa A, Giralt J, Pelegrín L, SánchezDalmau B, Segura A, García-Arumí J, et al. Ocular syphilis--back again: understanding recent increases in the incidence of ocular syphilitic disease. Ocul Immunol Inflamm 2009; 17 (3): 207-12.

10.- Ishihara S. Tests for colour-blindness (Handaya, Tokyo, Hongo Harukicho, 1917).

11.- Deschenes J, Seamone C D, Baines M G. Acquired ocular syphilis: diagnosis and treatment. Ann Ophthalmol 1992; 24 (4): 134-8.

12.- Becerra L I, Ksiazek S M, Savino P J, Marcus D K, Buckley R M, Sergott RC, et al. Syphilitic uveitis in human immunodeficiency virus-infected and noninfected patients. Ophthalmology 1989; 96 (12): 1727-30.

13.- Chao J R, Khurana R N, Fawzi A A, Reddy H S, Rao N A. Syphilis: reemergence of an old adversary. Ophthalmology 2006; 113 (11): 2074-9.

14.- Lasso M B, Balcells M E, Fernández A S, Gaete P G, Serri M V, Pérez J G, et al. Neurosyphilis in the patients with and without HIV infection: description and comparison of two historical cohorts. Rev Chil Infectol 2009; 26 (6): 540-7.

15.- Müller M, Ewert I, Hansmann F, Tiemann C, Hagedorn HJ, Solbach W, et al. Detection of Treponema pallidum in the vitreous by PCR. Br J Ophthalmol 2007; 91 (5): 592-5.

16.- Rajan M S, Pantelidis P, Tong C Y, French G L, Graham E M, Stanford M R. Diagnosis of Treponema pallidum in vitreous samples using real time polymerase chain reaction. Br J Ophthalmol 2006; 90 (5): 647-8.

17.- Seña A C, White B L, Sparling P F. Novel Treponema pallidum serologic tests: a paradigm shift in syphilis screening for the 21 st century". Clin Infect Dis 2010; 51: 700-8.

18.- American Academy of Ophthalmology. The Eye M.D. Association. Intraocular inflammation and uveitis. 2007-2008. p. 276.

19.- Goh B T, van Voorst Vader P C; European Branch of the International Union Against Sexually Transmitted Infection and the European Office of the World Health Organization. European guideline for the management of syphilis. Int J STD AIDS 2001; 12 Suppl 3: 14-26.

20.- Centers for Disease Control and Prevention. Trends in Reportable Sexually Transmitted Diseases in the United States. CDC, National Report. 2004.

21.- Stoner B P. Current controversies in the management of adult syphilis. Clin Infect Dis 2007; 44 Suppl 3: S130-46.

22.- Centers for Disease Control and Prevention. Sexually Transmitted Diseases Treatment Guidelines, 2006. MMWR Recomm Rep 2006; 55 (RR-11): 1-94.

23.- Solensky R. Hypersensivity reactions to betalactam antibiotics. Clin Rev Allergy Immunol 2003; 24 (3): 201-20. 
24.- Ghanem K G, Erbelding E J, Cheng W W, Rompalo A M. Doxycycline compared with benzathine penicillin for the treatment of early syphilis. Clin Infect Dis 2006; 42 (6): e45-9.

25.- Wong T, Singh A E, De P. Primary syphilis: serological treatment response to doxycycline/ tetracycline versus benzathine penicillin. Am J Med 2008; 121 (10): 903-8.

26.- Bai Z G, Yang K H, Liu Y L, Tian J H, Ma B, Mi D H, et al. Azithromycin vs. benzathine penicillin $\mathrm{G}$ for early syphilis: a meta-analysis of randomized clinical trials. Int J STD AIDS 2008; 19 (4): 217-21.

27.- Shalaby I A, Dunn J P, Semba R D, Jabs D A. Syphilitic uveitis in human immunodeficiency virus-infected patients. Arch Ophthalmol 1997; 115 (4): 469-73.
28.- Browning D J. Posterior segment manifestations of active ocular syphilis, their response to a neurosyphilis regimen of penicillin therapy, and the influence of human immunodeficiency virus status on response. Ophthalmology 2000; 107 (11): 2015-23.

29.- Margo C E, Hamed L M. Ocular syphilis. Surv Ophthalmol 1992; 37 (3): 203-20.

30.- Stoumbos V D, Klein M L. Syphilitic retinitis in a patient with acquired immunodeficiency syndrome-related complex. Am J Ophthalmol 1987; 103 (1): 103-4.

31.- Fu E X, Geraets R L, Dodds E M, Echandi L V, Colombero D, McDonald H R, et al. Superficial retinal precipitates in patients with syphilitic retinitis. Retina 2010; 30 (7): 1135-43.
32.- McLeish W M, Pulido J S, Holland S, Culbertson W W, Winward K. The ocular manifestations of syphilis in the human immunodeficiency virus type 1-infected host. Ophthalmology 1990; 97 (2): 196-203.

33.- Blocker M E, Levine W C, St Louis M E. HIV prevalence in patients with syphilis, United States. Sex Transm Dis 2000; 27 (1): 53-9.

34.- Reddy S, Cubillan L D, Hovakimyan A, Cunningham ET Jr. Inflammatory ocular hypertension syndrome (IOHS) in patients with syphilitic uveitis. Br J Ophthalmol 2007; 91 (12): 1610-2.

35.- Thompson H S, Kardon R H. The Argyll Robertson pupil. J Neuroophthalmol 2006; 26 (2): 134-8. 